\title{
Combination of FGFR4 inhibitor Blu9931 and 5-fluorouracil effects on the biological characteristics of colorectal cancer cells
}

\author{
DONGBAO JIANG $^{1 *}$, JINGJING LI $^{2 *}$, JIE LI $^{1}$, MIN WANG $^{3}, \mathrm{CHAO} \mathrm{HAN}^{4}$, \\ XINRU WANG ${ }^{2}$, CHUNLIN ZHAO $^{1}$ and YANWEI YE ${ }^{1}$
}

\author{
Departments of ${ }^{1}$ Gastrointestinal Surgery and Institute of Clinical Medicine, and ${ }^{2}$ Gastroenterology, \\ The First Affiliated Hospital of Zhengzhou University; ${ }^{3}$ Department of Function, \\ The Fifth Affiliated Hospital of Zhengzhou University; ${ }^{4}$ Department of Pharmacy, \\ The First Affiliated Hospital of Zhengzhou University, Zhengzhou, Henan 45000, P.R. China
}

Received August 11, 2017; Accepted September 26, 2017

DOI: $10.3892 /$ ijo.2017.4143

\begin{abstract}
The aim of this study was to explore the effects of single agent treatments and combination of Blu9931 and 5-fluorouracil (5-FU) on the biological characteristics of colorectal cancer cells and its mechanism. Blu9931 is the first selective small molecule inhibitor of the fibroblast growth factor receptor 4 (FGFR4) and exquisitely selective for FGFR4 versus other FGFR family members and all other kinases. The colorectal cancer cells HCT116 and SW620 with high expression of FGFR4 were selected for a series of functional tests including cell viability, cell proliferation, apoptosis and cell cycle detection. Western blotting was used to detect the expression of related molecules including signal pathway (STAT3), apoptosis (cleaved caspase-3), cell cycle (cyclin D1 and P27 ${ }^{\mathrm{kipl}}$ ) and epithelial-mesenchymal transition (E-cadherin and vimentin) in HCT116 and SW620 cells used as single and combination treatments of 5-FU and Blu9931. The cell viability gradually decreased when the concentration of 5-FU and Blu9931 increased. Blu9931 can inhibit FGFR4 protein expression while 5-FU cannot, as assessed by western blot analysis. The single agent treatment and combinations of 5-FU and Blu9931 arrest cell cycle $(\mathrm{P}<0.05)$, increased p27 $7^{\mathrm{kipl}}$ expression and reduced cyclin D1 expression. The single agent
\end{abstract}

Correspondence to: Dr Yanwei Ye, Department of Gastrointestinal Surgery and Institute of Clinical Medicine, The First Affiliated Hospital of Zhengzhou University, 1 Eastern Jian-She Road, Zhengzhou, Henan 450052, P.R. China

E-mail: yeyanwei66@126.com

*Contributed equally

Abbreviations: CRC, colorectal cancer; 5-FU, 5-fluorouracil; FGFR, fibroblast growth factor receptor; FGFR4, fibroblast growth factor receptor 4

Key words: Blu9931, 5-FU, FGFR4, FGFR, colorectal cancer, in vivo, proliferation, apoptosis, epithelial-mesenchyman transition treatment and combinations of 5-FU and Blu9931 inhibited EMT. Furthermore, the combination of 5-FU and Blu9931 has a synergistic effect in reducing colorectal cancer cell proliferation and preventing cell cycle. Taken together, this study provides the first evidence that Blu9931 functions as a FGFR4-selective inhibitor in colorectal cancer (CRC) cells, and Blu9931 may be a new targeted drug.

\section{Introduction}

Colorectal cancer (CRC) is the second most common cause of cancer death in the Western world (1), and the incidence of colorectal cancer in the Asia-Pacific region is increasing (2). Surgical resection of primary tumors and adjuvant chemotherapy improved patient survival, but nearly half of the patients eventually died of local recurrence and metastasis (3). At present, targeted therapy has become an important treatment for various malignant tumors (including CRC). Bevacizumab (targeted to VEGF) and cetuximab (targeted to EGFR) are two drugs approved for use in the treatment of progressive and/or metastatic colorectal cancer. Molecular targeted therapy also has the problem of multi-target combination therapy.

Now, exploring new therapeutic targets in colorectal cancer has become an essential goal. The fibroblast growth factor receptor (FGFRs) is a polygene family, an immunoglobulin gene superfamily member, which is distributed in a variety of cells on the membrane protein. FGFR4 is a class of transmembrane tyrosine kinase receptors with autophosphorylation activity that plays a very important role in embryonic development, tissue repair and angiogenesis (4). Hagel et al discovered Blu9931, which is a novel irreversible kinase inhibitor that specifically targets FGFR4 and is also the first FGFR4-selective molecule for the treatment of patients with hepatocellular carcinoma (5).

Our previous studies have shown that the expression of FGFR4 mRNA is significantly increased in gastric cancer tissue when compared with that of the corresponding normal tissue (6). The knockdown of FGFR4 expression resulted in a decrease in the proliferation of MKN45 and SGC7901 GC cell lines and an increase in apoptosis. Western blot analysis showed that the expression of caspase- 3 was increased and 
the expression of Bcl-xL in MKN45 and SGC7901 cells was decreased after FGFR4-siRNA transfection (7). The apoptosis rates were obviously increased in GC cells treated with the single and combination of 5-FU and PD173074 (an inhibitor of FGFR) (8). Nonetheless we have not yet studied the role of FGFR4 in colorectal cancer.

In order to investigate the clinical value of FGFR4 expression in CRC and explore new targeted drugs, Blu9931 as a specific inhibitor of FGFR4 was introduced in this study. We selected two colorectal cancer cell lines, HCT116 and SW620, that overexpress FGFR4. A series of functional tests were then performed to study the effects of single agent treatment and combinations of 5-FU and Blu9931 on the biological behavior of the above two cell lines, including proliferation assay, apoptosis detection, cell cycle distribution assessment and the expression of related molecules by western blot analysis. Epithelial-mesenchymal transition (EMT) is considered a key event in metastasis and plays a critical role in the progression of colorectal cancer. Ectopic FGF19 expression promotes EMT and invasion in epithelial-like HCC cells by inhibiting of E-cadherin expression (9). FGF19 interacts with FGFR4 and promotes FGFR4 expression. We found that FGFR4 and E-cadherin expression are negatively correlated in colorectal cancer cells. Through these functional tests, our aim is to elucidate the mechanism of Blu9931 and 5-FU on colorectal cancer cells. We believe that FGFR4 is a potential therapeutic target for colorectal cancer and that Blu9931 may be used in the treatment of colorectal cancer patients.

\section{Materials and methods}

Cell lines and cell culture. Human colorectal cancer cell lines LS47 and SW620 were purchased from the American Type Culture collection. DLD1, SW116 and HCT116 cell lines were purchased from the Chinese Academy of Sciences, the Science Cell Bank of the Type Culture Collection (CBTCCCAS, Shanghai, China). Cell lines were cultivated in Dulbecco's modified Eagle's medium (DMEM, Sweden) supplemented with $10 \%$ fetal bovine serum (FBS; Gibco, USA), $100 \mathrm{U} / \mathrm{ml}$ of penicillin and $100 \mu \mathrm{g} / \mathrm{ml}$ of streptomycin (Caisson Labs, UT, USA) at $37^{\circ} \mathrm{C}$ in a humidified atmosphere containing $5 \% \mathrm{CO}_{2}$.

Antibodies and reagents. Rabbit monoclonal anti-FGFR4 antibody, anti-E-cadherin, anti-vimentin, anti-cleaved caspase-3, anti-STAT3, anti-cyclin D1, anti-p27 ${ }^{\mathrm{kip} 1}$ and anti$\beta$-actin antibody as well as mouse monoclonal anti-PCNA antibody were all purchased from Cell Signaling Technology (Beverly, MA, USA). Secondary horseradish peroxidaseconjugated antibodies were goat anti-mouse and goat anti-rabbit from Sigma-Aldrich Corp. (St. Louis, MO, USA). Blu9931 (HY-12823) was purchased from Shanghai Haoyuan Chemexpress (Shanghai, China). 5-FU was from the clinical trial group in our research center.

Reverse transcription PCR and quantitative real-time PCR. According to the protocol, TRIzol reagents are used to collect total RNA from colorectal cancer cell lines. The first strand cDNA synthesis kit (MBI, Fermentas, Canada) was reverse transcribed into cDNA for each RNA sample. The primers were: FGFR-4, 5'-AGATGCTCAAAGACAACGCCT-3' and 5'-CGCACTCCACGATCACGTA-3'; $\beta$-actin, 5'-CACGAT GGAGGGGCCGGACTCATC-3' and 5'-TAAAGACCTCTA TGCCAACACAGT -3 '. The 2X Taq PCR MasterMix (Tiangen Biotech, China) was used for PCR. The FGFR4 annealing temperature was $57^{\circ} \mathrm{C}$. The PCR product was subjected to $2 \%$ agarose gel electrophoresis and stained with ethidium bromide. Gene-specific primers of FGFR4 and GAPDH were the same as the reverse transcriptase PCR in our study. Five colorectal cancer cell lines were examined by real-time PCR according to the specification by Takara (Japan). The experiment was carried out in duplicate. Relative differences (-fold) were calculated according to the comparative $\mathrm{Ct}$ method.

Protein extraction and western blotting. Whole-cell lysates were prepared using the Mammalian Protein Extraction reagent (Merck, Darmstadt, Germany) in accordance with the manufacturer's instructions. Protein concentrations of the samples were determined by the bicinchoninic acid (BCA) protein assay (Pierce, Rockford, IL, USA). Protein samples (30 $\mu \mathrm{g}$ of each protein) boiled for $5 \mathrm{~min}$ were separated on $10 \%$ SDS-polyacrylamide gels and transferred onto PVDF membranes. The membranes were blocked for $1 \mathrm{~h}$ at room temperature with phosphate-buffered saline (PBS) containing $0.05 \%$ Tween-20 and 5\% non-fat dried milk, and incubated overnight at $4^{\circ} \mathrm{C}$ with the primary antibodies following the manufacturer's instructions. Immunoblots were washed three times with PBS containing 0.05\% Tween-20 and $1 \%$ non-fat milk. Then PVDF membranes incubated with secondary antibodies conjugated with horseradish peroxidase against mouse $\mathrm{IgG}$ or rabbit $\mathrm{IgG}$ for $1 \mathrm{~h}$ at room temperature. Immunoreactive proteins were using the ECL detection system (ImageQuant LAS 3000; General Electric Co., Fairfeld, CT, USA). Three independent western blot assays were performed for all samples.

Cell viability assay. HCT116 cells and SW620 cells in logarithmic growth phase were harvested, and cell density was adjusted to $3 \times 10^{5} / \mathrm{ml}$. Then, these cells were seeded into 96-well plates $(100 \mu \mathrm{l} /$ well $)$ and there were 4 wells in each group. After 24-h incubation, the cells were treated with single and combination of Blu9931 and 5-FU at different concentrations. According to Blu9931 $(0,1.875,3.75,7.5,15$ and $30 \mu \mathrm{M})$ and $5-\mathrm{FU}(0,3.125,6.25,12.5,25$ and $50 \mu \mathrm{M})$ were studied. Cells were incubated at $37^{\circ} \mathrm{C}$ in an environment of $5 \% \mathrm{CO}_{2}$ for $72 \mathrm{~h}$. After addition of $5 \mathrm{mg} / \mathrm{ml}$ MTT $(10 \mu \mathrm{l} / \mathrm{well})$, cells were incubated for $4 \mathrm{~h}$ and the supernatant was removed. DMSO (100 $\mu \mathrm{l} /$ well) was added to each well and cells were further incubated for $10 \mathrm{~min}$ with constant shaking to resolve purple crystals. The absorbance was measured at $490 \mathrm{~nm}$ using a microplate reader. The cell viability and $\mathrm{IC}_{50}$ were calculated and analyzed.

Proliferation assay. HCT116 cells and SW620 cells in logarithmic growth phase were harvested, and cell density was adjusted to $2 \times 10^{5} / \mathrm{ml}$. Then, these cells were seeded into 96-well plates $(100 \mu \mathrm{l} /$ well $)$ and there were 4 wells in each group. After 24-h incubation, the cells were treated with single and combination of Blu9931 and 5-FU at $\mathrm{IC}_{50}$. After culturing for 1, 2, 3, 4 and 5 days, cells were incubated for 

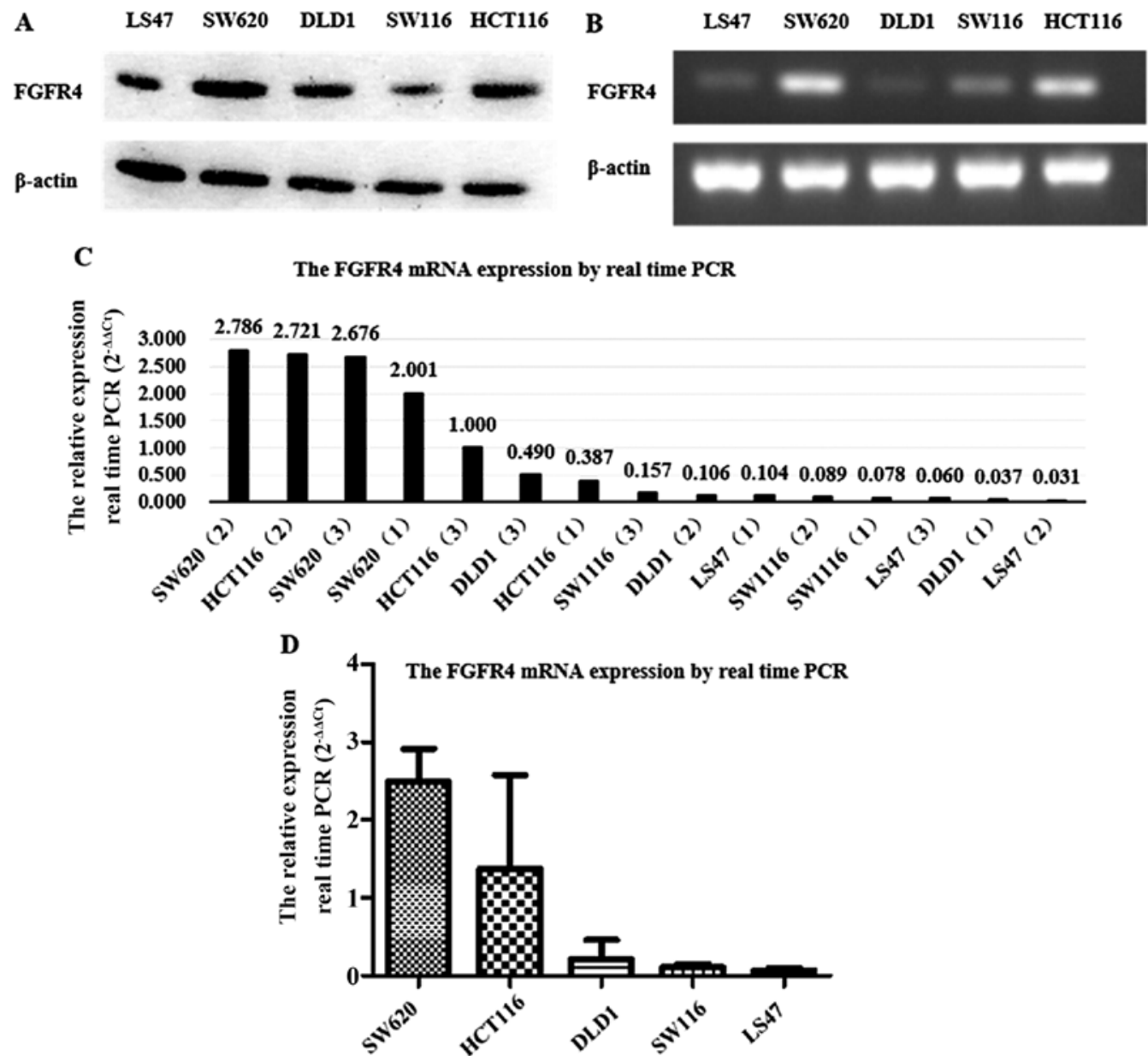

Figure 1. The expression of FGFR4 mRNA and protein in five colorectal cancer cell lines. (A) Expression of FGFR4 protein in various colorectal cancer cell lines by western blotting. (B) Expression of FGFR4 mRNA in various colorectal cancer cell lines by RT-PCR. (C andD) Expression of FGFR4 mRNA in various colorectal cancer cell lines by quantitative real-time PCR. $\beta$-actin s served as loading control. The expression of FGFR4 mRNA and protein in HCT116 cells and SW620 cells was higher than that in the other three colorectal cancer cell lines. At least three independent evaluations were performed.

$4 \mathrm{~h}$ with $5 \mathrm{mg} / \mathrm{ml}$ MTT (10 $\mu \mathrm{l} /$ well) and the supernatant was removed. DMSO (100 $\mu \mathrm{l} /$ well $)$ was added to each well and cells were further incubated for $10 \mathrm{~min}$ with constant shaking to resolve purple crystals. The absorbance was measured at $490 \mathrm{~nm}$ using a microplate reader.

Apoptosis rate detection. HCT116 cells and SW620 cells were treated with single and combination of Blu9931 and 5-FU at each proper concentration for $72 \mathrm{~h}$. Then the cells were harvested. Annexin V and APC were used to detect apoptosis by flow cytometry. Cells were suspended in $500 \mu 1$ of Annexin $\mathrm{V}$ binding buffer and the cells were incubated with $5 \mu \mathrm{l}$ of Annexin $\mathrm{V}$ for $15 \mathrm{~min}$ in the dark and at room temperature. Then $5 \mu 1$ of propidium iodide was added. Thereafter, all samples were analyzed by a FACSCalibur (BD Bioscience) flow cytometer with CellQuest software.

Cell cycle analysis. HCT116 cells and SW620 cells were treated with single and combination of Blu9931 and 5-FU at each proper concentration for $72 \mathrm{~h}$. The cells were then collected and centrifuged at $1,000 \mathrm{rpm}$ for $5 \mathrm{~min}$. The supernatant was discarded and the pre-cooled PBS was added to wash the cells twice. Then $70 \%$ pre-cooled ethanol was added, overnight at $4^{\circ} \mathrm{C}$. The ethanol-immobilized cells were centrifuged, the supernatant was discarded and cells washed three times with precooled PBS. The cells were resuspended using $1 \mathrm{ml}$ of PI/ Triton X-100 staining solution (20 $\mu \mathrm{g}$ PI/0.1\% Triton X-100) containing $0.2 \mathrm{mg}$ RNase A and stained at $37^{\circ} \mathrm{C}$ for $15 \mathrm{~min}$. Samples were placed on ice and immediately analysed on Beckman Coulter to separate G0/G1, S, G2/M and hypodiploid nuclei. All assays were carried out in triplicate.

Statistical analysis. Statistical analysis was carried out with SPSS 13.0 software (USA). Data were showed by means \pm SD and three individual experiments were performed. Student's t-test was used to compare data between two groups. One-way ANOVA and Tukey's test were applied to compare data between three or more groups. $\mathrm{P}<0.05$ was considered statistically significant.

\section{Results}

FGFR4 expression is different in various colorectal cell lines. FGFR4 was expressed in colorectal cancer cell lines at mRNA and protein levels using reverse transcription PCR, real-time PCR and western blot analysis. As Fig. 1A illustrates, FGFR4 

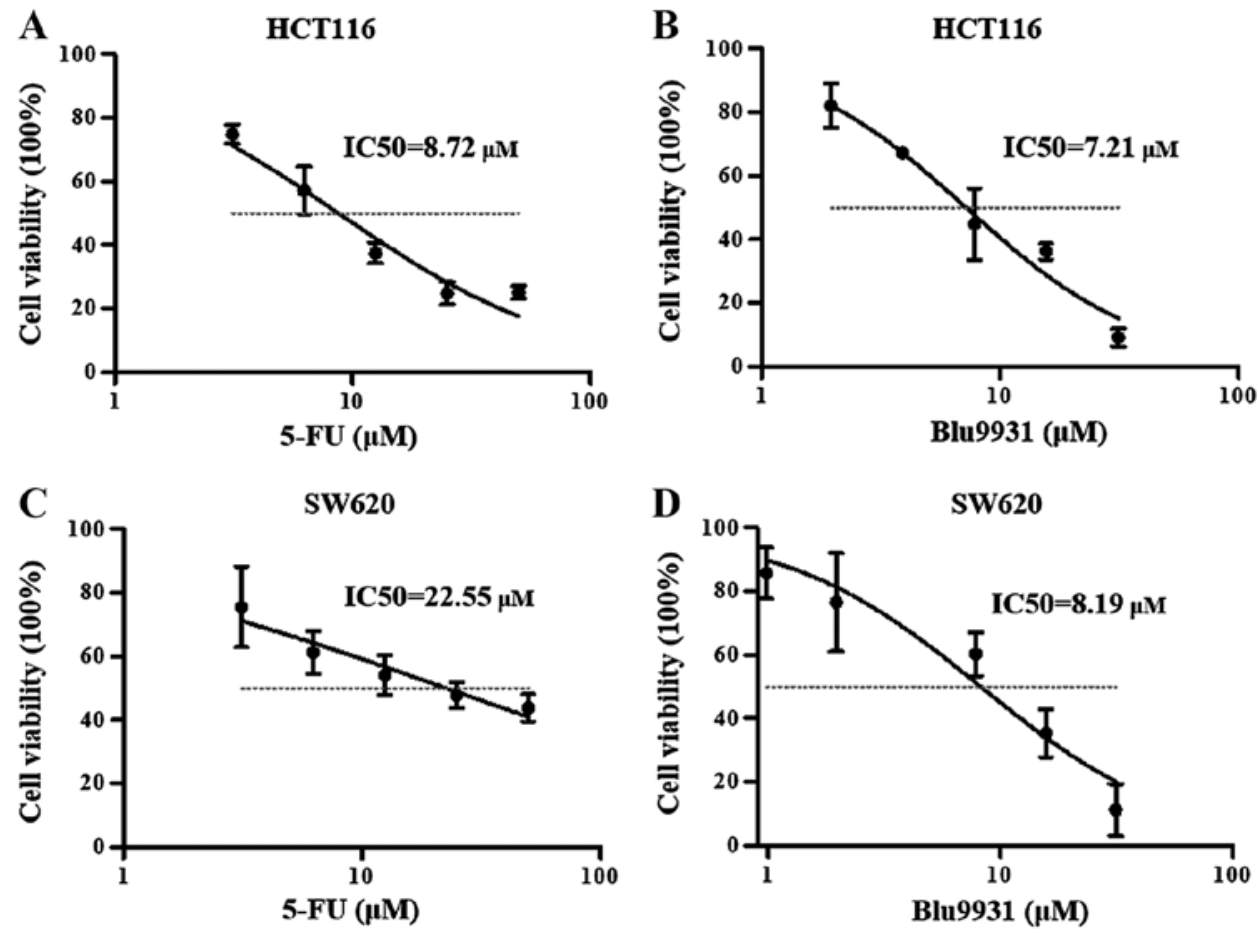

Figure 2. Effects on the cell viability of HCT116 and SW620 cells of the different reagents using MTT. With the increase of concentration of 5-FU (A) and Blu9931 (B), the cell viability of HCT116 cells decreased. Similarly, with the increase in the concentration of the two reagents, SW620 cell viability also decreased $(C$ and $D)$.

protein expression was stronger in HCT116 and SW620, while weaker in LS47, DLD1 and SW116. Reverse transcription PCR results showed that FGFR4 mRNA expression was significantly higher in HCT116 and SW620 than in the other three colorectal cancer cell lines (Fig. 1B), which was verified by quantitative real-time PCR $\left(2^{-\Delta \Delta c t}\right)$ through at least three independent detectors (Fig. 1C). As shown in Fig. 1D, the quantitative analysis results by real-time PCR confirmed that FGFR4 mRNA expression in HCT116 and SW620 were much higher than the other colorectal cancer lines. Therefore, the HCT116 and SW620 cell lines were chosen to undergo subsequent assays.

5-FU and Blu9931 have an effect on the cell viability of HCT116 and SW620 cells. In order to evaluate the growth effect of different reagents on HCT116 and SW620 cells, MTT assays were used to detect the cell viability using a microplate reader. Fig. $2 \mathrm{~A}$ and $\mathrm{B}$ showed that the concentration of 5-FU and Blu9931 increased, the cell viability of HCT116 cells progressively decreased, the $\mathrm{IC}_{50}$ of 5-FU and Blu9931 were 8.72 and $7.21 \mu \mathrm{M}$, respectively. When 5-FU and Blu9931 acted on SW620 cells, the cell viability also decreased with increasing concentration, and $\mathrm{IC}_{50}$ of 5-FU and Blu9931 were 22.55 and $8.19 \mu \mathrm{M}$, respectively (Fig. 2C and D). For subsequent analysis, the appropriate concentration of each agent is selected in the linear region. According to the above results, in HCT116 cells, the appropriate concentration of 5-FU and Blu9931 is $10 \mu \mathrm{M}$. In SW620 cells, the appropriate concentrations of 5-FU and Blu9931 are $20 \mu \mathrm{M}$ and $10 \mu \mathrm{M}$, respectively.

Single agent treatments and the combination of 5-FU and Blu9931 affect the proliferation of HCT116 and SW620 cells.
In order to explore whether FGFR4 is a therapeutic target for colorectal cancer, we have introduced FGFR4-specific inhibitor Blu9931 and 5-FU to intervene in HCT116 and SW620 cells. MTT assay was used to detected cell absorbance to evaluate cell proliferation.

The single and combination of 5-FU and Blu9931 obviously weakened the proliferative ability of HCT116 and SW620 cells when compared with its corresponding Mock group (Fig. 3A and B; one-way ANOVA; $\mathrm{P}<0.05$ ). As shown in Fig. 3C, the proliferative ability of the Blu9931 group was slightly stronger than that of the 5-FU group on days 1,3 and 5 in HCT116 cells (Student's t-test; $\mathrm{P}<0.05$ ). However, the proliferation ability had no significant difference between the 5-FU group and Blu9931 group SW620 cells (Fig. 3D; Student's t-test; $\mathrm{P}<0.05)$. Compared with Blu9931 group, the proliferation ability in HCT116 cells treated with the combination of 5-FU and Blu9931 significantly decreased from day 2 to day 5 while it significantly decreased in SW620 cells from day 3 to day 5 after MTT assay (Fig. 3E and F; Student's t-test; $\mathrm{P}<0.05)$. Compared with 5-FU group, the proliferation ability significantly decreased in HCT116 and SW620 cells treated with the combination of 5-FU and Blu9931 from day 1 to day 5 after MTT assay (Fig. 3G and H; Student's t-test; P<0.05).

Single agent treatment and the combination of 5-FU and Blu9931 affect the apoptosis rate of HCT116 and SW620 cells. To study whether the different reagents affect apoptosis of the colorectal cancer cells, the apoptotic rate of HCT116 and SW620 cells was detected by flow cytometry and treated with the single agent treatment and the combination of 5-FU and Blu9931 for $72 \mathrm{~h}$. In both HCT116 cells and SW620 cells, the apoptotic rate of each treatment group increased, 

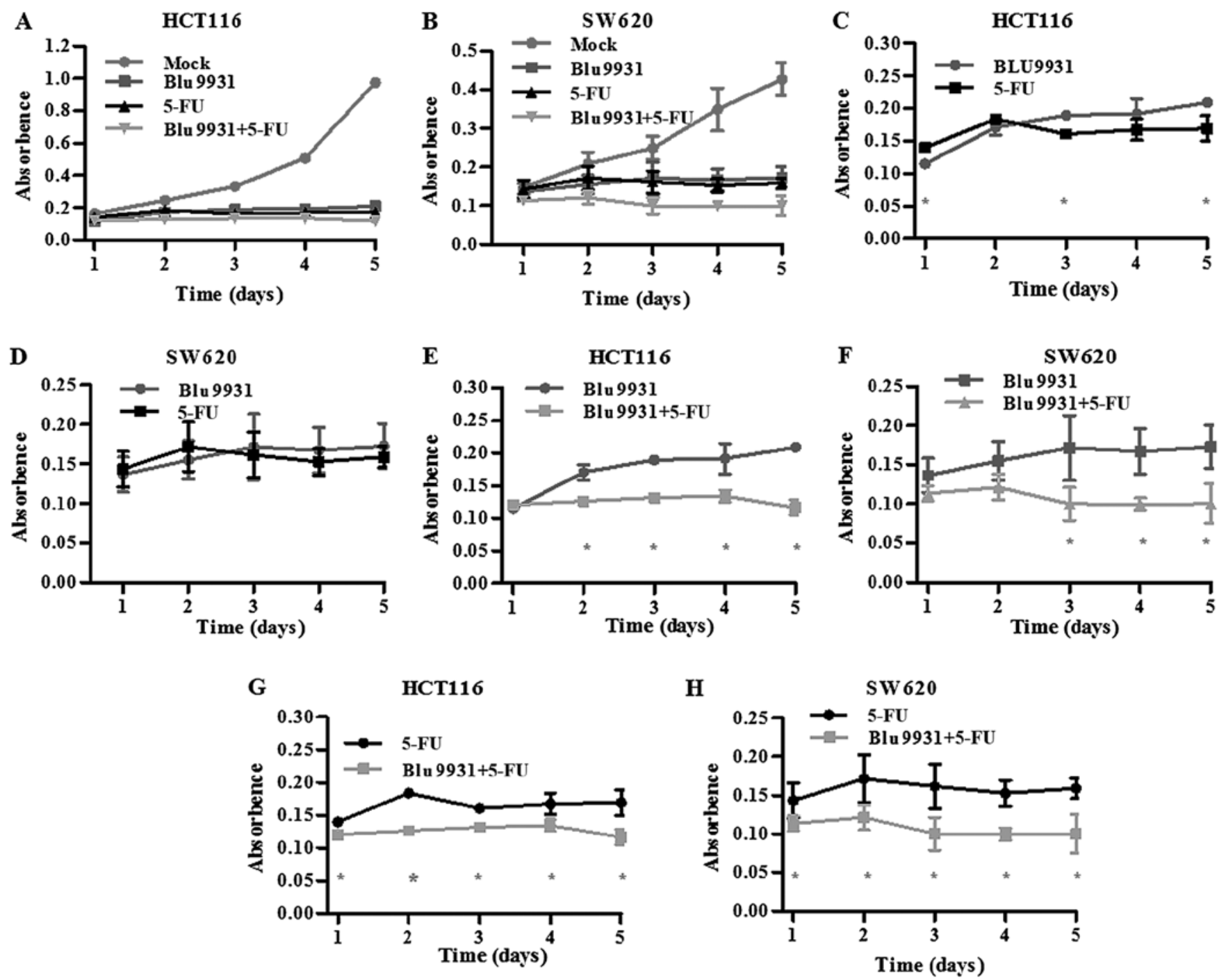

Figure 3. Influence of single and combination of 5-FU and Blu9931 on the proliferation of colorectal cancer cell lines as detected by MTT. Cell absorbance was consecutively measured at 5 days. (A and B) The single and combination of 5-FU and Blu9931 obviously weakened the proliferative ability of HCT116 and SW620 cells. (C and D) There was no significant difference between the Blu9931 group and 5-FU group (one-way ANOVA; $\left.{ }^{*} \mathrm{P}<0.05\right)$. (E and F) The combination of 5-FU and Blu9931 obviously weakened the proliferative ability of HCT116 and SW620 cells when compared with the Blu9931 group (Student's t-test, $\left.{ }^{*} \mathrm{P}<0.05\right)$. ( $\mathrm{G}$ and H) The combination of 5-FU and Blu9931 obviously weakened the proliferative ability of HCT116 and SW620 cells when compared with the 5-FU group (Student's t-test, ${ }^{*} \mathrm{P}<0.05$ ).

the difference was statistically significant (Fig. 4; one-way ANOVA; P<0.05). However, in HCT116 cells, the apoptosis rate of 5-FU plus Blu9931 was higher than that in the single of 5-FU and Blu9931 (one-way ANOVA; P<0.05). However, there was no statistically significant difference among 5-FU, Blu9931 and 5-FU plus Blu9931 in SW620 cells (one-way ANOVA; $\mathrm{P}<0.05)$.

Single agent treatment and the combination of 5-FU and Blu9931 affect cell cycle distribution of HCT116 and SW62O cells. The distribution of cell cycle was evaluated by flow cytometry in HCT116 and SW620 cells with the single and combined treatment of Blu9931 and 5-FU. As Fig. 5A-E shows, the HCT116 cells treated with the single and combined Blu9931 and 5-FU in S stage evidently increased while those in G0/G1 stage remarkably decreased compared to the mock group (one-way ANOVA; $\mathrm{P}<0.05$ ). However, in SW620 cells, the $\mathrm{G} 2 / \mathrm{M}$ stage cells in the three treatment groups were much less than those in the mock group. The SW620 cells treated with the single Blu9931 in S stage significantly increased while 5-FU group and 5-FU plus Blu9931 group remarkably increased in G0/G1 stage (Fig. 5F-J; one-way ANOVA; $\mathrm{P}<0.05)$.

Single agent treatment and the combination of 5-FU and Blu9931 suppress EMT in HCT116 and SW620 cells. The epithelial-mesenchymal transition (EMT) plays an important role in tumor invasion and metastasis. In the EMT process, the cell phenotype transforms from epithelial into mesenchymal, and cell markers also change (10). The expression of E-cadherin was decreased and the expression of vimentin was increased. To investigate whether the different reagents affect EMT in the colorectal cancer cells, we detected the expression of E-cadherin and vimentin by western blotting. In HCT116 cells, expression of E-cadherin was increased in the single and combination of 5-FU and Blu9931 compared with control 

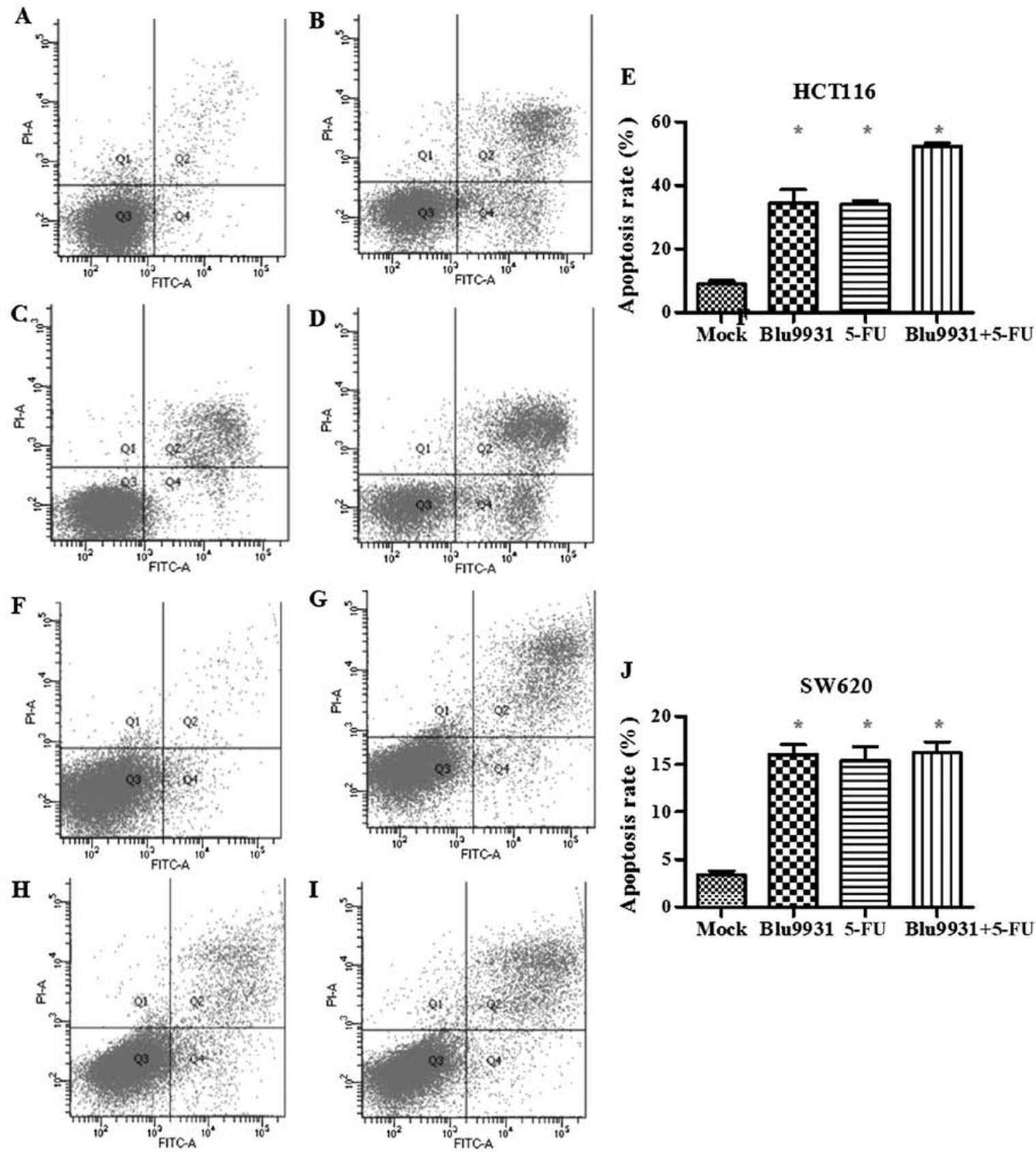

Figure 4. The effects of single and combined treatment of Blu9931 and 5-FU on the apoptosis rate of HCT116 and SW620 cells were evaluated by flow cytometry. (A-D) Apoptosis of HCT116 treated with control. (A) Mock group; (B) 5-FU group; (C) Blu9931 group; (D) 5-FU plus Blu9931. (E) In HCT116 cells, the apoptosis rate of single and combination of 5-FU and Blu9931 was significantly higher than that in the control group (one-way ANOVA; P<0.05). (F-I) Apoptosis of SW620 treated with control. (F) Mock group; (G) 5-FU group; (H) Blu9931 group; (I) 5-FU plus Blu9931. (J) In SW620 cells, the apoptosis rate of single and combination of 5-FU and Blu9931 was significantly higher than that in the control group (one-way ANOVA; P<0.05).

group while vimentin expression was weakened. The same result was seen in SW620 cells. The above results show that Blu9931 and 5-FU can suppress EMT (Fig. 6A and B).

Single agent treatment and the combination of 5-FU and Blu9931 affect the expression of related molecules in HCT116 and SW620 cells. Western blotting was used to detect the expression of related molecules including signal pathway (STAT3), apoptosis (cleaved caspase-3), and cell cycle (cyclin D1 and P27 ${ }^{\mathrm{kip} 1}$ ) in HCT116 and SW620 cells when treated by the single and combination of 5-FU and Blu9931. As shown in Fig. 6C and D, expression of FGFR4 in HCT116 and SW620 cells was decreased in the single Blu9931 group and the combination of 5-FU and Blu9931 group while no significant difference was noted in the single 5-FU group when compared with the control group. When compared with the control group, expression of STAT3 was markedly increased in the single agent 5-FU group. Compared to the control group, cleaved caspase-3 expression in HCT116 and SW620 cells was obviously increased in the single and combination of 5-FU and Blu9931. In other words, the single and combination of 5-FU and Blu9931 had an effect on promoting apoptosis, but 5-FU plus Blu9931 group was no significant difference in the single agent 5-FU and single Blu9931 treatment groups. 
A

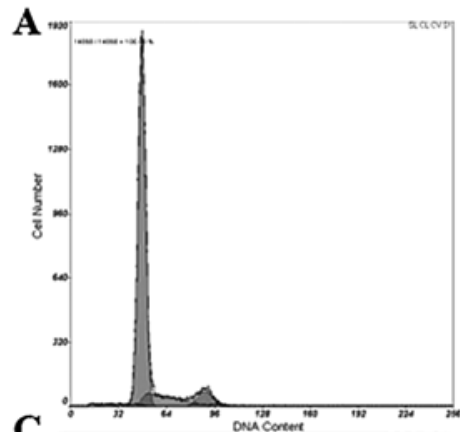

$\mathrm{C}_{\text {. }}$

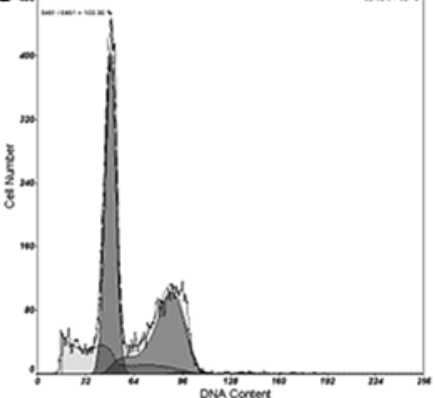

F

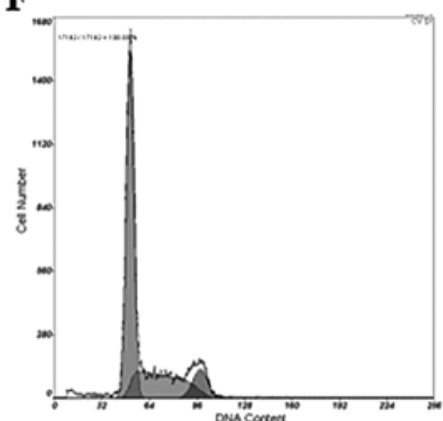

H



B

D
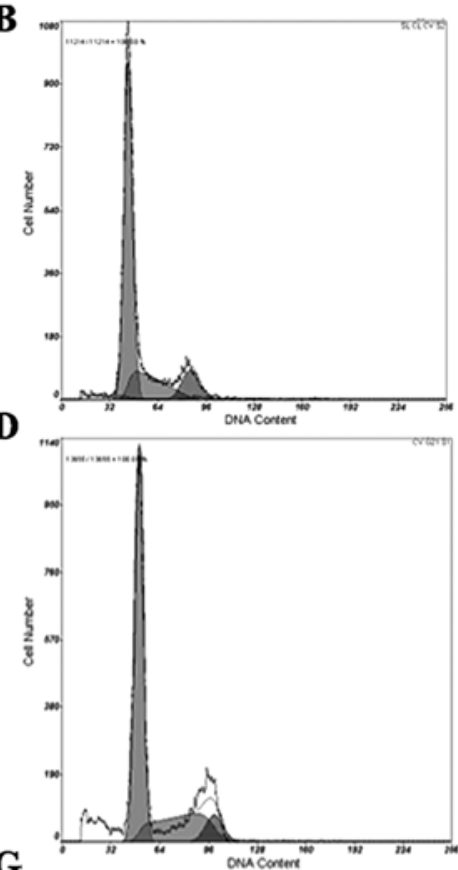

G

I

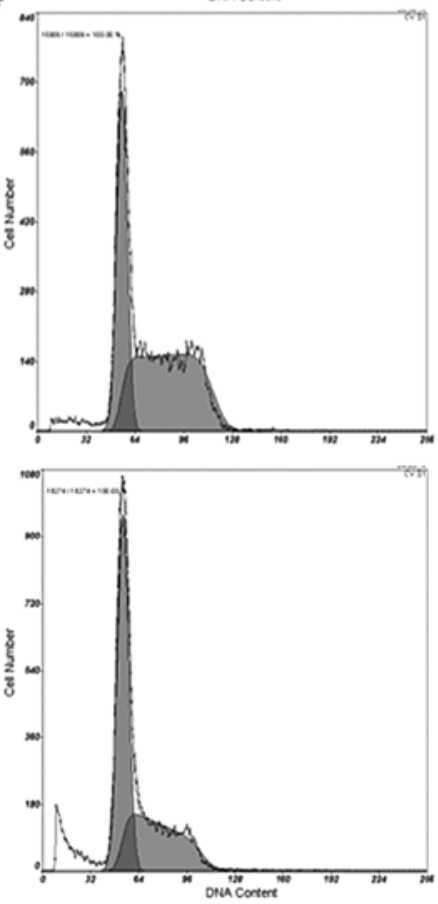

E
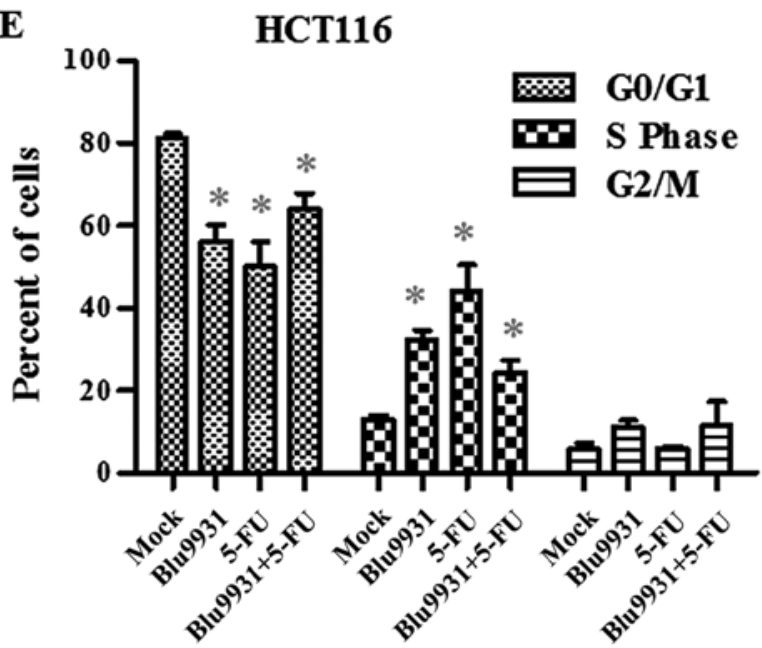

J

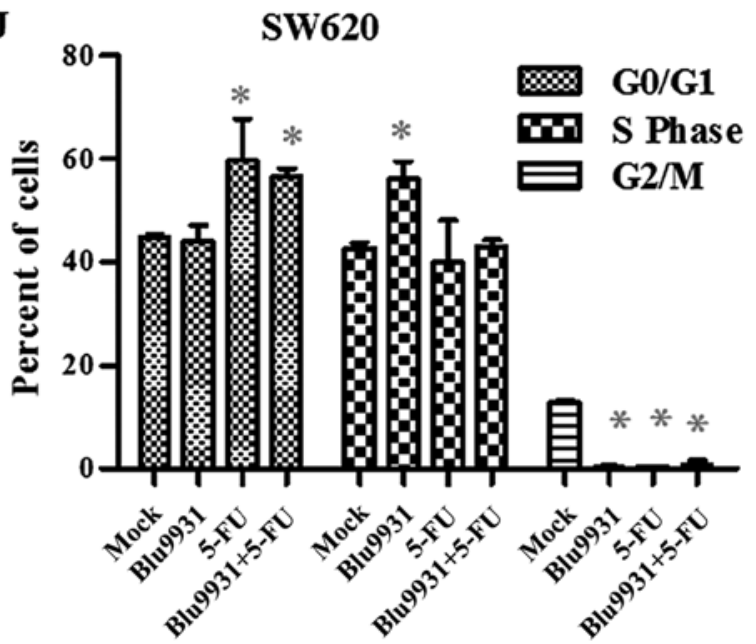

Figure 5. The distribution of cell cycle was evaluated by flow cytometry in HCT116 and SW620 cells with the single and combined treatment of Blu9931 and 5-FU. (A-E) Cell cycle distribution of HCT116 cells with different treatments. (A) Mock group; (B) Blu9931 group; (C) 5-FU group; (D) Blu9931 plus 5-FU group. (E) The $\mathrm{S}$ stage cells in the three treatment groups were significantly higher than those in mock group (one-way ANOVA; $<<0.05$ ). (F-J) Cell cycle distribution of SW620 cells with different treatments. (F) Mock group; (G) Blu9931 group; (H) 5-FU group; (I) Blu9931 plus 5-FU group. (J) The G2/M stage cells in the three treatment groups were much less than those in mock group (one-way ANOVA; $\mathrm{P}<0.05$ ). All data shown are representative of at least three independent experiments.

When compared to the control group, $\mathrm{P} 27^{\mathrm{kip} 1}$ expression was obviously increased in the single and combination of 5-FU and Blu9931 while cyclin D1 expression was prominently weakened.

\section{Discussion}

With the development of tumor molecular biology, the study of tumor molecular targeted therapy has become the current hot spot. Molecular targeted drugs have also made some progress in the application of colorectal cancer. A number of phase II clinical studies have shown that cetuximab (anti-EGFR) has a high efficacy $(11,12)$ in the treatment of advanced colorectal cancer. However, the curative effect was not satisfactory. So exploring new therapeutic targets in CRC has become an essential goal. Katoh (13) suggested that the FGFR family may be important in clinical cancer diagnostics and therapeutics. FGFR4 plays a very important role in embryonic develop- 
A

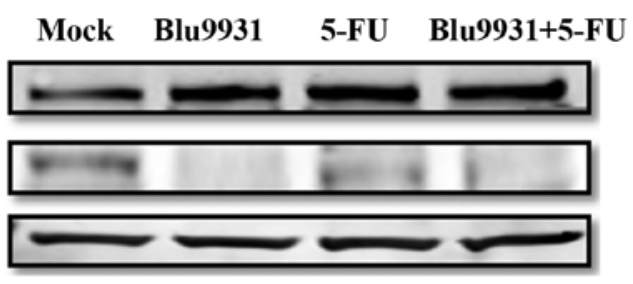

C

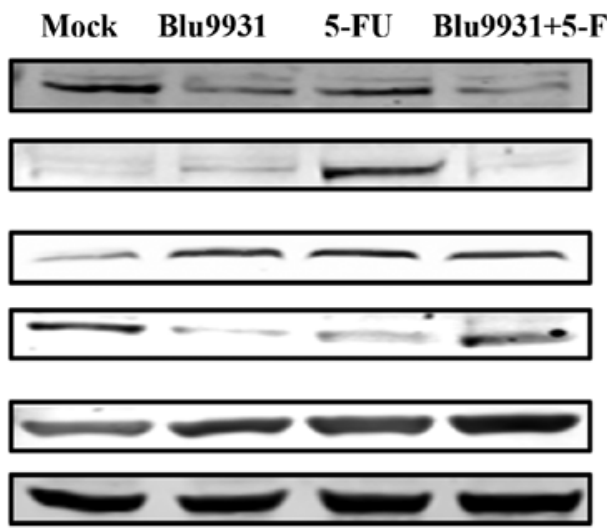

B

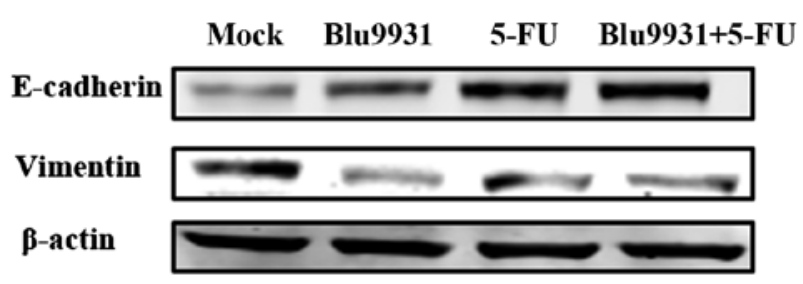

D

SW620

FGFR4



STAT3
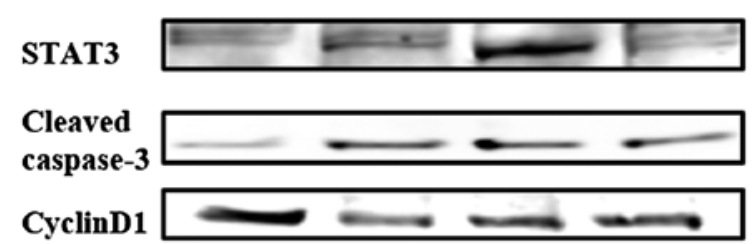

P27 ${ }^{\text {kip } 1}$

B-actin



Figure 6. Western blot detection of the expression of related molecules including signal pathway (STAT3), apoptosis (cleaved caspase-3), cell cycle (cyclin D1 and P27 ${ }^{\mathrm{kipl}}$ ) and epithelial-mesenchymal transition (E-cadherin and vimentin) in HCT116 and SW620 cells when treated by the single and combination of 5-FU and Blu9931. (A and C) Expression of related molecules in HCT116 cells when treated by control, 5-FU, Blu9931 and 5-FU plus Blu9931. (B and D) Expression of related molecules in SW620 cells when treated by control, 5-FU, Blu9931 and 5-FU plus Blu9931.

ment, tissue repair and angiogenesis and has become a popular research molecule in a variety of tumors. However, the effect of FGFR4 in CRC has rarely been studied.

There are only two reports associated with Blu9931, both of which are not associated with colorectal cancer. Furthermore, $5-\mathrm{FU}$ is a cell cycle-specific chemotherapeutic agent that is primarily resistant to cells in the $\mathrm{S}$ phase. In this study, we reported that the treatment of Blu9931 significantly reduced cell viability and increased cell apoptosis rates when compared with the negative control group. The effects of the combination treatment of 5-FU and Blu9931 in proliferation and arresting cell cycle were superior to that of the single agent treatments.

As shown in Fig. 1, in five common colorectal cancer cell lines, the expression of FGFR4 at mRNA and protein levels in HCT116 and SW620 cells was significantly higher than the other three cells. Thus, HCT116 and SW620 cells were chosen for the subsequent assays. With the concentration of 5-FU and Blu9931 increasing, the cell viability of HCT116 and SW620 cells gradually decreased after administration as single agents. This finding shows that both Blu9931 and 5-FU can significantly reduce the viability of colorectal cancer cells. Hagel et al showed Blu9931 markedly inhibited the viability of hepatocellular cancer cells $\left(\mathrm{EC}_{50}<1 \mu \mathrm{M}\right)(5)$.

The single and combination of 5-FU and Blu9931 obviously weakened the proliferative ability of HCT116 and SW620 cells when compared with its corresponding Mock group (Fig. 3). Moreover, proliferation ability of the combination treatment of 5-FU and Blu9931 was significantly less than that in the single agent treatments. It suggests that the combination of 5-FU and Blu9931 had synergistic effects on the inhibition of proliferation rates in CRC cells. As shown in Fig.6, expression of FGFR4 in the HCT116 and SW620 cells was decreased in the single Blu9931 group and the combination of 5-FU and Blu9931 group while no significant difference was noted in the single 5-FU group when compared with the control group. Therefore, Blu9931 mainly reduced the proliferation of CRC cells by inhibiting the activity of FGFR4, whereas 5-FU was not. Bai et al (14) indicated that FGFR4 inhibitor PD173074 obviously suppressed colon cancer cell proliferation and neovascularization. Also, in embryonal rhabdomyosarcoma, inhibition of FGFR4 expression reduces cell proliferation in vitro and xenograft formation in vivo (15).

In both HCT116 and SW620 cells, the apoptosis rate of single agent treatment and combinations of 5-FU and Blu9931 increased when compared with the control group. Only in HCT116 cells, the apoptosis rate of 5-FU plus Blu9931 was higher than that in the single of 5-FU and Blu9931. Also, western blot analysis showed that the single and combination of 5-FU and Blu9931 obviously increased cleaved caspase-3 expression, and 5-FU plus Blu9931 group had no significant difference in the single agent 5-FU and single Blu9931 treatment groups in HCT116 and SW620 cells. In other words, the single agent treatment 5-FU and Blu9931 can induce colorectal cancer cell apoptosis, but Blu9931 plus 5-FU does not seem to make the apoptosis rate higher. Blu9931-induced apoptosis may be mediated by decreasing FGFR4 expression. Our previous studies have shown that silencing FGFR4 can increase tumor cell apoptosis rates (7). Zaid et al (16) indicated that the downregulation of FGFR4 significantly prevented the WNT pathway, suggesting that WNT may be 
one of the signaling pathways that affect apoptosis. Only in the single agent treatment 5-FU, stat3 expression was significantly elevated relative to the negative control group and the other two treatment groups. This result is very interesting, because usually high expression of stat 3 promotes cell growth and reduces the rate of apoptosis. We hypothesized that 5-FU may induce STAT3 expression, whereas STAT3 is essential for lysosomal pathways that induce cell death (17).

Our study also investigated the effects of different treatments on cell cycle distribution in HCT116 and SW620 cells by flow cytometry. HCT116 cells treated with the single and combined of Blu9931 and 5-FU in S stage evidently increased while those in G0/G1 stage remarkably decreased compared to mock group, which indicated that cell cycle was blocked in the S phase. SW620 cells treated with the single Blu9931 arrested in the S stage while 5-FU group and 5-FU plus Blu9931 group arrested in the G0/G1 stage. The antitumor effect of 5-FU is mainly mediated by inhibition of thymidylate synthase (TS) and its incorporation metabolites into RNA and DNA (18). Twenty-four-hour exposure to 5-FU produced S-phase accumulation in MCF-7 breast cancer cells (19). However, Chong et al showed that 5-FU-induced cell cycle arrested at the G0/G1 phases in SW480 and HT29 cells (20). Now, we are not particularly aware of the mechanism of 5-FU on the cell cycle. However, we can see that 5-FU has different effects on human tumor cell cycle. The western blot results also demonstrated that the cell cycle was inhibited. When the P27 protein expression increased, the most critical cell cycle protein E-CDK2 complex activity was inhibited, and cell cycle stagnated in $\mathrm{G} 1$ phase $(21,22)$. Cyclin D1 plays an important role in the development of tumor as an important positive regulator of the cell cycle (23-25). In this study, P2 $7^{\mathrm{kip} 1}$ expression remarkably strengthened in the three treatment groups compared to the negative control while cyclin D1 observably weakened in the HCT116 and SW620 cells. Moreover, the P27 ${ }^{\mathrm{kip} 1}$ expression following the combination treatment of 5-FU and Blu9931 was stronger than that in the single agent treatments which suggesting that the combination of 5-FU and Blu9931 had synergistic effects on the inhibition of the cell cycle.

EMT plays a key role in the development, progression, invasion and metastasis of malignant tumors. There are EMT phenomena in the process of malignancy and invasion of peripheral tissues (26). Brabletz et al (27) found that inhibition of E-cadherin expression, induced $\beta$-catenin nuclear translocation can enhance the ability of colorectal cancer cell invasion, and low expression of E-cadherin is associated with distant metastasis of colorectal cancer. Zhao et al (28) showed that FGFR4 overexpression inhibited E-cadherin expression by activation of GSK3 $\beta / \beta$-catenin pathway and knocked it down to produce an opposite effect in hepatocellular carcinoma cells. Our findings suggest that Blu9931 as a specific inhibitor of FGFR4 can inhibit EMT in colorectal cancer cells. Inhibition of FGFR4 expression can promote the expression of E-cadherin and reduce the expression of vimentin.

In conclusion, this study explored the effects of single agent treatments and combination of Blu9931 and 5-FU on the biological characteristics of colorectal cancer cells and its mechanism. Inhibiting the activity of FGFR4 may be one of the principal mechanisms of Blu9931 to inhibit the proliferation of colorectal cancer cells, increase the apoptosis rate, prevent the cell cycle and inhibit EMT. The combination of 5-FU and Blu9931 has a synergistic effect in reducing colorectal cancer cell proliferation and preventing the cell cycle. The results of this study further demonstrate that the first specific inhibitor of FGFR4, Blu9931, can be a novel target drug for the treatment of colorectal cancer. Obviously, our results should be validated by further studies in vitro and in vivo.

\section{Acknowledgements}

This study was supported by Department of Gastrointestinal Surgery and Institute of Clinical Medicine, The First Affiliated Hospital, Zhengzhou University and National Natural Science Foundation of China, grant no. 81201955.

\section{Reference}

1. Longley DB and Johnston PG: Molecular mechanisms of drug resistance. J Pathol 205: 275-292, 2005.

2. Kimman M, Norman R, Jan S, Kingston D and Woodward M: The burden of cancer in member countries of the Association of Southeast Asian Nations (ASEAN). Asian Pac J Cancer Prev 13: 411-420, 2012.

3. Punt CJ and Tol J: More is less - combining targeted therapies in metastatic colorectal cancer. Nat Rev Clin Oncol 6: 731-733, 2009.

4. Eswarakumar VP, Lax I and Schlessinger J: Cellular signaling by fibroblast growth factor receptors. Cytokine Growth Factor Rev 16: 139-149, 2005.

5. Hagel M, Miduturu C, Sheets M, Rubin N, Weng W, Stransky N, Bifulco N, Kim JL, Hodous B, Brooijmans N, et al: First selective small molecule inhibitor of FGFR4 for the treatment of hepatocellular carcinomas with an activated FGFR4 signaling pathway. Cancer Discov 5: 424-437, 2015.

6. Packer LM and Pollock PM: Paralog-specific kinase inhibition of FGFR4: Adding to the arsenal of anti-FGFR Agents. Cancer Discov 5: 355-357, 2015

7. Ye YW, Zhou Y, Yuan L, Wang CM, Du CY, Zhou XY, Zheng BQ, Cao X, Sun MH, Fu H, et al: Fibroblast growth factor receptor 4 regulates proliferation and antiapoptosis during gastric cancer progression. Cancer 117: 5304-5313, 2011.

8. Li J, Ye Y, Wang M, Lu L, Han C, Zhou Y, Zhang J, Yu Z, Zhang X, Zhao C, et al: The over-expression of FGFR4 could influence the features of gastric cancer cells and inhibit the efficacy of PD173074 and 5-fluorouracil towards gastric cancer. Tumour Biol 37: 6881-6891, 2016.

9. Sawey ET, Chanrion M, Cai C, Wu G, Zhang J, Zender L, Zhao A, Busuttil RW, Yee H, Stein L, et al: Identification of a therapeutic strategy targeting amplified FGF19 in liver cancer by Oncogenomic screening. Cancer Cell 19: 347-358, 2011.

10. Jang MJ, Baek SH and Kim JH: UCH-L1 promotes cancer metastasis in prostate cancer cells through EMT induction. Cancer Lett 302: 128-135, 2011.

11. Dfaz Rubio E, Tabernero J, van Cutsem E, Cavantes A and Andre T: Hu-Inblet Y, Soulie P, Corretge S, Kisker O and de Gramont A: Cetuximab in combination with oxaliplatin/5fluorouracil(5-FU)/folinic acid(FA)(FoLFoX-4) in the first-1ine treatment of patients with epidermal growth factor receptor EGFR)-expressing metastatic colorectal cancer: An international Phase II study. J Clin Oncol 23: S16, 2005.

12. Folprecht G, Lutz MP, Schöffski P, Seufferlein T, Nolting A, Pollert P and Köhne CH: Cetuximab and irinotecan/5-fluorouracil/ folinic acid is a safe combination for the first-line treatment of patients with epidermal growth factor receptor expressing metastatic colorectal carcinoma. Ann Oncol 17: 450-456, 2006.

13. Katoh M: Genetic alterations of FGF receptors: An emerging field in clinical cancer diagnostics and therapeutics. Expert Rev Anticancer Ther 10: 1375-1379, 2010.

14. Bai YP, Shang K, Chen H, Ding F, Wang Z, Liang C, Xu Y, Sun MH and Li YY: FGF-1/-3/FGFR4 signaling in cancer-associated fibroblasts promotes tumor progression in colon cancer through Erk and MMP-7. Cancer Sci 106: 1278-1287, 2015.

15. Crose LE, Etheridge KT, Chen C, Belyea B, Talbot LJ, Bentley RC and Linardic CM: FGFR4 blockade exerts distinct antitumorigenic effects in human embryonal versus alveolar rhabdomyosarcoma. Clin Cancer Res 18: 3780-3790, 2012. 
16. Zaid TM, Yeung TL, Thompson MS, Leung CS, Harding T, Co NN, Schmandt RS, Kwan SY, Rodriguez-Aguay C, Lopez-Berestein $\mathrm{G}$, et al: Identification of FGFR4 as a potential therapeutic target for advanced-stage, high-grade serous ovarian cancer. Clin Cancer Res 19: 809-820, 2013.

17. Wake MS and Watson CJ: STAT3 the oncogene - still eluding therapy? FEBS J 282: 2600-2611, 2015.

18. Longley DB, Harkin DP and Johnston PG: 5-fluorouracil: Mechanisms of action and clinical strategies. Nat Rev Cancer 3: $330-338,2003$

19. Grem JL, Nguyen D, Monahan BP, Kao V and Geoffroy FJ: Sequence-dependent antagonism between fluorouracil and paclitaxel in human breast cancer cells. Biochem Pharmacol 58 : 477-486, 1999

20. Chong D, Ma L, Liu F, Zhang Z, Zhao S, Huo Q, Zhang P, Zheng H and Liu H: Synergistic antitumor effect of 3-bromopyruvate and 5-fluorouracil against human colorectal cancer through cell cycle arrest and induction of apoptosis. Anticancer Drugs 28: 831-840, 2017.

21. Nourse J, Firpo E, Flanagan WM, Coats S, Polyak K, Lee MH Massague J, Crabtree GR and Roberts JM: Interleukin-2mediated elimination of the $\mathrm{p} 27^{\mathrm{Kipl}}$ cyclin-dependent kinase inhibitor prevented by rapamycin. Nature 372: 570-573, 1994.

22. Peng D, Fan Z, Lu Y, DeBlasio T, Scher H and Mendelsohn J: Anti-epidermal growth factor receptor monoclonal antibody 225 up-regulates $\mathrm{p} 27^{\mathrm{KIP} 1}$ and induces G1 arrest in prostatic cancer cell line DU145. Cancer Res 56: 3666-3669, 1996.
23. Zhou JX, Niehans GA, Shar A, Rubins JB, Frizelle SP and Kratzke RA: Mechanisms of G1 checkpoint loss in resected early stage non-small cell lung cancer. Lung Cancer 32: 27-38, 2001.

24. Moghaddam SJ, Haghighi EN, Samiee S, Shahid N, Keramati AR, Dadgar S and Zali MR: Immunohistochemical analysis of p53, cyclin D1, RB1, c-fos and N-ras gene expression in hepatocellular carcinoma in Iran. World J Gastroenterol 13: 588-593, 2007.

25. Rose SL and Buller RE: The role of p53 mutation in BRCA1associated ovarian cancer. Minerva Ginecol 54: 201-209, 2002.

26. Li Y, Wang W, Wang W, Yang R, Wang T, Su T, Weng D, Tao T, Li W, Ma D, et al: Correlation of TWIST2 up-regulation and epithelial-mesenchymal transition during tumorigenesis and progression of cervical carcinoma. Gynecol Oncol 124: 112-118, 2012.

27. Braumbtz T, Jung A, Reu S, Porzner M, Hlubek F, Leoni A, Kunz S, Knuechel R and Kirchner T: Variable beta-catenin expression in colorectal cancers indicate tumor progression driven by the tumor environment. Proc Natl Acad Sci USA 98: 10356-10361, 2001.

28. Zhao H, Lv F, Liang G, Huang X, Wu G, Zhang W, Yu L, Shi L and Teng Y: FGF19 promotes epithelial-mesenchymal transition in hepatocellular carcinoma cells by modulating the GSK3 $3 / \beta$ catenin signaling cascade via FGFR4 activation. Oncotarget 7 : 13575-13586, 2016. 